\title{
Non-Contact Vibration Monitoring of Power Transmission Belts Through Electrostatic Sensing
}

\author{
Yonghui Hu, Yong Yan, Fellow, IEEE, Lijuan Wang, and Xiangchen Qian
}

\begin{abstract}
On-line vibration monitoring plays an important role in the fault diagnosis and prognosis of industrial belt drive systems. This paper presents a novel measurement technique based on electrostatic sensing to monitor the transverse vibration of power transmission belts in an on-line, continuous, and non-contact manner. The measurement system works on the principle that variations in the distance between a strip-shaped electrode and the naturally electrified dielectric belt give rise to a fluctuating current output. The response of the sensor to a belt moving both axially and transversely is numerically calculated through finite-element modeling. Based on the sensing characteristics of the sensor, the transverse velocity of the belt is characterized through the spectral analysis of the sensor signal. Experiments were conducted on a two-pulley belt drive system to verify the validity of the sensing technique. The belt vibration at different axial speeds was measured and analyzed. The results show that the belt vibrates at well-separated modal frequencies that increase with the axial speed. A closer distance between the electrode and the belt makes higher order vibration modes identifiable, but also leads to severer signal distortion that produces higher order harmonics in the signal.
\end{abstract}

Index Terms-Belt drive, vibration monitoring, electrostatic sensor, finite element modelling, sensing characteristics.

\section{INTRODUCTION}

B ELT drives are extensively used in many industries to transmit power between rotational machine elements. Common applications include engine accessory drive in automobiles, spindle drive in CNC (Computer Numerical Control) machines, carriage drive in printers and drum drive in washing machines. In comparison with chains, gears and linkages, belt drives are advantageous in terms of cost, maintenance requirement, misalignment tolerance, operation smoothness and shock absorption. However, the compliance of the flexible belt makes it more susceptible to large amplitude vibrations that cause noise radiation, fatigue failure and incorrect

Manuscript received January 22, 2016; accepted February 11, 2016. Date of publication February 15, 2016; date of current version April 8, 2016. This work was supported in part by the National Natural Science Foundation of China under Grant 51375163 and Grant 61573140 and in part by the Ministry of Education, China, under Grant B13009. The associate editor coordinating the review of this paper and approving it for publication was Dr. Joseph Shor.

$\mathrm{Y}$. $\mathrm{Hu}$ and X. Qian are with the School of Control and Computer Engineering, North China Electric Power University, Beijing 102206, China (e-mail: huyhui@gmail.com; xcqian@live.com).

Y. Yan is with the School of Engineering and Digital Arts, University of Kent, Kent CT2 7NT, U.K. (e-mail: y.yan@kent.ac.uk).

L. Wang is with the School of Control and Computer Engineering, North China Electric Power University, Beijing 102206, China, and also with the School of Engineering and Digital Arts, University of Kent, Canterbury CT2 7NT, U.K. (e-mail: wwlljj2007@126.com).

Digital Object Identifier 10.1109/JSEN.2016.2530159 functionality of the mechanical system. Since some common operation abnormalities such as belt defects, imbalance, wear and resonance are usually associated with belt vibration, on-line monitoring and characterization of belt vibration could serve as an effective and reliable tool for the fault diagnosis and failure prognosis of the machinery [1].

The objective of this research was to develop a novel measurement technique based on electrostatic sensing for on-line, continuous and non-contact monitoring of belt vibration. The electrification of dielectrics is a ubiquitous phenomenon in the industries. By making good use of this phenomenon, the electrostatic sensors have been used to achieve a variety of monitoring and diagnostic objectives, such as flow characterization of particles in pneumatic conveying pipelines [1], [2] and gas-solid fluidized beds [3], [4], detection of gas path debris for health monitoring of an aero-engine [6], [7], condition monitoring and failure prediction of a taper roller bearing [8], and speed measurement of rotating shafts [9] and running strips [10]. Recent studies have found that a power transmission belt moving both axially and transversely generates a fluctuating electric field that can be detected using an electrostatic sensor [11]. Since the fluctuation of the sensor signal was mainly attributed to the transverse vibration of the belt, characterization of the belt transverse displacement was achieved through the analysis of the normalized spectra of the sensor signal. In this paper, a detailed study of the sensing mechanism and fundamental characteristics of the electrostatic sensor for vibration monitoring of power transmission belts was carried out. Moreover, the performance of the measurement system was experimentally assessed under a wider range of conditions. A comparative analysis of the belt vibration kinematics and the sensor response reveals that the belt transverse velocity can be represented by the sensor signal. It is verified that the velocity spectrum of the multimode vibration can be measured using the electrostatic sensing technique.

\section{Previous Related Work}

The vibration of power transmission belt has been investigated for many years but still remains a challenging subject today [12]. A belt-drive system, consisting of a flexible belt, multiple fixed pulleys and a spring-loaded tensioner, can exhibit highly dynamical and complex vibratory behaviors arising from a variety of sources. Hitherto, the majority of studies on belt vibration have focused on theoretical modelling 
TABLE I

Comparison of the Techniques CurRently AVAilable for Belt Vibration Monitoring

\begin{tabular}{lll}
\hline \hline \multicolumn{1}{c}{ Techniques } & \multicolumn{1}{c}{ Advantages } & Disadvantages \\
\hline Digital imaging [23] & Non-contact measurement & Computationally expensive for on-line measurement \\
& Highly intuitive & Difficult to use in dusty and extreme environments \\
\hline Laser Doppler vibrometer [24-26] & Non-contact measurement & Prohibitively expensive to implement in routine industrial applications \\
& Wide bandwidth & Too delicate to use in dusty, harsh and extreme environments \\
& High sensitivity & \\
& High spatial resolution & Unable to measure absolute vibration parameters \\
\hline Electrostatic sensing & Non-contact measurement & \\
& Simple structure & \\
& Easy installation & \\
& Low cost & \\
& Suitability for harsh environments & \\
\hline \hline
\end{tabular}

and numerical analysis to predict the dynamic response of the belt systems [13]-[17], and there is an obvious lack of research on measurement techniques for on-line monitoring and diagnosis purposes.

The measurement of transverse vibration of an axially moving belt is a particularly difficult problem. Conventional contact type sensors such as accelerometers and strain gauges cannot be used due to the impractical requirement of physical contact with the moving surface [18]-[20]. Several types of proximity sensors based on eddy current, ultrasonic, capacitive or inductive principles are often used to measure the relative vibration of rotating shafts [21], [22], but their applications to belt vibration measurement are difficult for reasons related to measurement range, installation requirement, and material property of the object being measured. A high-speed camera, able to capture 500 frames per second, was used by Catalano et al. [23] for vibration analysis of a belt system. This technique is non-contact, non-invasive and highly intuitive; however, on-line measurement cannot be realized due to the high computational cost required to accurately locate the marker on the belt in each frame. A well-established technique for non-contact vibration measurement is Laser Doppler Vibrometer (LDV) working on the principle of laser interferometry. Sante and Rossi [24] measured the operating deflection shapes (ODS) of a synchronous belt using a scanning LDV for development of a vibro-acoustic model. Agnani et al. [25] experimentally investigated the dynamic behavior of a V-belt system by means of a single-point LDV that measures the transverse belt vibration and a scanning LDV that measures the out-of-plane velocity at multiple discrete positions along the belt span. Moon and Wickert [26] studied nonlinear vibration of a belt system, which is excited by pulleys having slight eccentricity, by measuring the transverse belt displacement and velocity using a laser interferometer. The laserbased technique has advantages of non-contact measurement, wide bandwidth, high sensitivity and high spatial resolution; however, such instruments are prohibitively expensive to implement in routine industrial applications and the use of delicate optical components limits their applicability in dusty, harsh and extreme environments. Currently, due to the lack of a practical technique for vibration monitoring, diagnosis of the belt condition is usually undertaken via visual inspection, which is prone to error and requires the machine to be switched off.
The electrostatic sensing technique investigated in this paper achieves belt vibration monitoring through induction of static charges on a copper strip located in the proximity of the running belt. The electrostatic sensor is non-intrusive, simple in structure, easy to install, cost-effective and well suited for harsh environments, making it a viable solution to the belt diagnostic problem. However, absolute vibration parameters cannot be measured using the electrostatic sensor due to unpredictable amount of charge on the belt. Table I summarizes the advantages and limitations of the techniques that have been applied to monitor belt vibration.

\section{SENSING CHARACTERISTICS AND MeAsurement Method}

\section{A. Measurement Principles}

As early as the 1970s, it had been found that static charges could build up on the surface of dielectric belts running on earthed metal rollers through contact and frictional electrification [27]. Along with the belt, the static charges undergo a combined axial and transverse motion, creating a fluctuating electric field in space. An insulated metal electrode placed adjacent to the belt generates an induced current signal in response to the passage of nearby charges. By converting the current signal into a proportional voltage signal using an I-V converter, information about motion of the belt can be derived. Fig. 1 illustrates the principle of the electrostatic sensor. The feedback resistor in the I-V converter determines the transimpedance gain, while the feedback capacitor ensures stability through phase compensation [28].

According to [27], the amount of charge generated on the belt depends on a variety of factors including roller speed, material property of the belt, temperature and atmospheric humidity. For fixed operating and environmental conditions, the density of charge is mainly determined by surface roughness of the belt, suggesting that the charges are randomly distributed. If the belt moved only axially, the fluctuation of the random electrostatic signal could reflect the variation of the surface roughness. But in practice, the belt vibrates transversely and the non-uniformity of surface roughness along the belt is very small. It is therefore deduced that the fluctuation of the sensor signal is dominated by the variation in the distance between the belt and the electrode. The above supposition, which constitutes the physical foundation of 


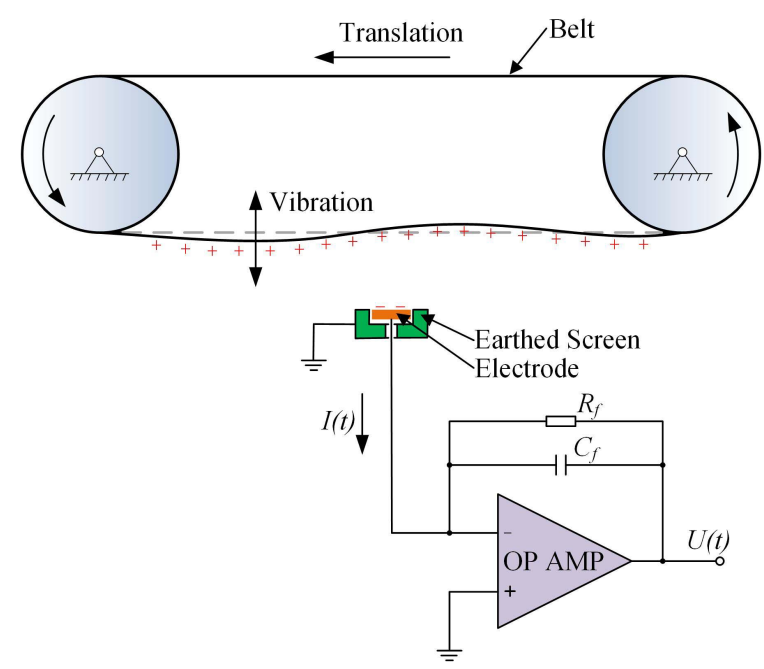

Fig. 1. Principle of the electrostatic sensor.

the measurement technique, will be experimentally validated in Section IV.

\section{B. Finite Element Modeling}

Electrostatic theories indicate that the amount of induced charge is inversely related to the distance between the belt and the electrode [29]. In order to obtain a quantitative analysis of the sensing characteristics, finite element modelling of the electrostatic sensor is carried out. In comparison with the theoretical modelling approach [30], finite element simulations provide efficient numerical solutions to the electrostatic boundary-value problem without solving any equations analytically.

A power transmission belt can be modelled as an axially moving string if its bending rigidity is negligible. Consider a string that travels at a constant transport speed $v$ between two supports separated by distance $L$. The dynamic equation that describes the transverse displacement $z(x, t)$ of the string at spatial coordinate $x$ and time $t$ is given by [4]

$\frac{\partial^{2} z}{\partial^{2} t}+2 v \frac{\partial^{2} z}{\partial t \partial x}-\left(c^{2}-v^{2}\right) \frac{\partial^{2} z}{\partial^{2} x}=0, \quad t>0$ and $0<x<L$

where $c$ is the wave speed due to a pretension of the string, and $c=\left(T_{0} / \mu\right)^{1 / 2}$ in which $T_{0}$ is the non-zero constant tension force and $\mu$ is the mass per unit length of the string. If the supports are fixed, the boundary conditions of the motion equation are given by

$$
z(0, t)=z(L, t)=0, \quad t \geq 0
$$

Solutions to equations (1) and (2) have been investigated using various analytical and numerical approaches in the literature [2]-[7]. For the sake of simplicity, the results obtained by Vermeer and Strasters [31] is adopted here. By assuming that the vibration modes of the string can be described by two sinusoidal waves propagating in opposite directions, the transverse deflection of the string is derived

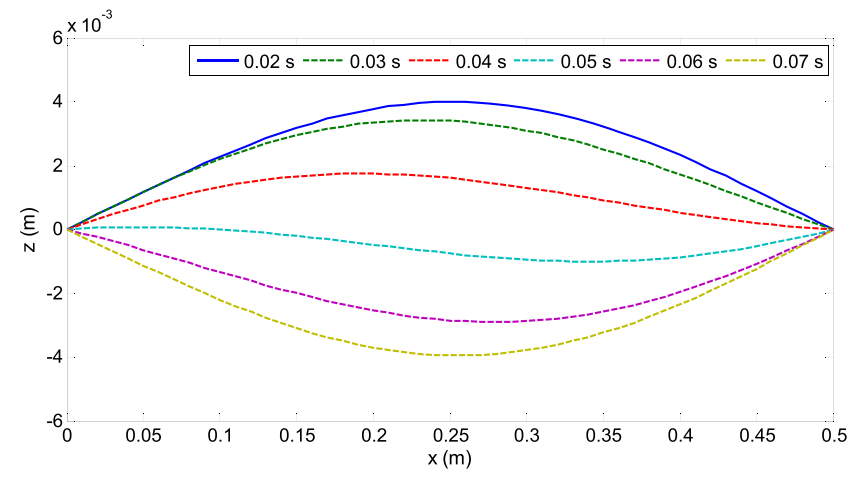

Fig. 2. The first mode shape in half a period of vibration.

as [31]

$$
z(x, t)=2 a \sin \left[\frac{n \pi}{L}\left(\frac{c^{2}-v^{2}}{c}\right)\left(t+\frac{v x}{c^{2}-v^{2}}\right)\right] \sin \frac{n \pi x}{L}
$$

where $a$ is the vibration amplitude of the sinusoidal wave, and $n$ is the mode number. Considering only the first vibration mode and letting $L$ be $0.5 \mathrm{~m}, v$ be $2 \mathrm{~m} / \mathrm{s}, c$ be $10 \mathrm{~m} / \mathrm{s}$ and $a$ be $0.002 \mathrm{~m}$, the curvatures of the belt in half a vibration period $(0.104 \mathrm{~s})$ is depicted in Fig. 2. As illustrated, the first vibration mode of the string resembles a standing wave, with only slight phase shifts along the belt span. It should be noted that in practice the belt displacement in the transverse direction is the superposition of all modes.

The finite element simulation of the induced charge on the electrode is based on the calculation of electric potential governed by the following Poisson equation

$$
\nabla(\varepsilon \nabla \Phi)=-\rho
$$

where $\Phi$ is the electric potential, $\rho$ is the charge density and $\varepsilon$ is the permittivity of the medium. Once $\Phi$ is solved, the induced charge $q^{\prime}$ on the electrode with surface area $S$ can be calculated through surface integration of the charge density

$$
q^{\prime}=\int_{S} \varepsilon \nabla \Phi d s
$$

Then the induced current flowing through the electrode is found as

$$
I=\frac{d q^{\prime}}{d t}
$$

The finite element model is constructed using the commercial software package COMSOL Multiphysics. The belt is modelled as an ideal surface with width of $20 \mathrm{~mm}$ and uniform charge density of $-1 \mu \mathrm{C} / \mathrm{m}^{2}$. The motion of the belt is given by the first vibration mode as shown in Fig. 2. The electrode is a thin copper strip with dimensions of $3 \mathrm{~mm}$ in width and $20 \mathrm{~mm}$ in length. The center of the electrode is located $6 \mathrm{~mm}$ below the center of the stationary belt. The medium of the computational region is air with a dielectric constant of 1 . The potential of the electrode is set as zero and the boundary of the model domain is set as zero charge. For accurate calculation of the induced charge, the maximum element size is set as $0.8 \mathrm{~mm}$ for the tetrahedral mesh elements with 


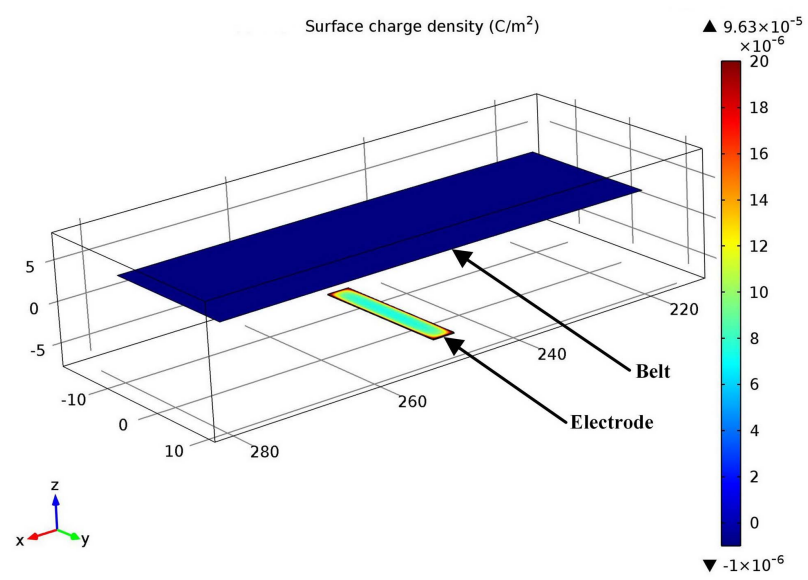

Fig. 3. Surface charge density on the electrode (the wireframe box indicates the computational region of the finite element model).
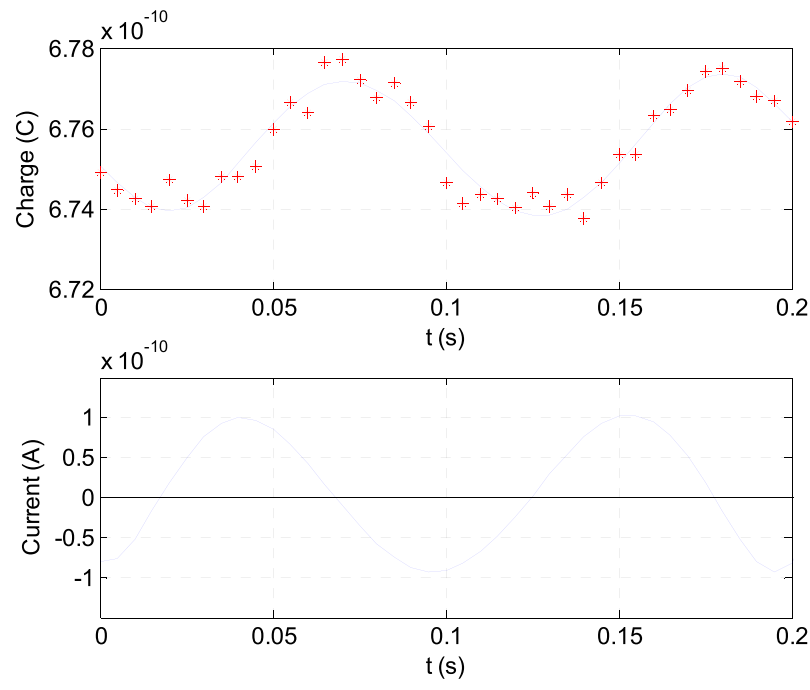

Fig. 4. Induced charge and current output due to belt vibration.

quadratic order of the geometry shape functions. In order to reduce the computational overhead, the belt elements out of the abscissa range from $0.22 \mathrm{~m}$ to $0.28 \mathrm{~m}$ are not taken into account since their contribution to the induced charge is negligible. Fig. 3 shows the simulated surface charge density on the electrode. As can be seen, the boundary areas are populated with more charges than the other areas due to the skin effect. Through surface integration, the total induced charge on the electrode is calculated as $0.674 \mathrm{nC}$.

The response of the electrostatic sensor to the motion of the belt is simulated using the parametric sweep function of the software. The temporal resolution is set as $0.005 \mathrm{~s}$ and the result is calculated for a total of $0.2 \mathrm{~s}$. The induced charge is expected to vary smoothly over time. However, due to the discretization error, the finite element simulation yields a rugged curve if the data points are connected directly. To overcome this problem, a curve fitting technique, using an eighth order polynomial, is applied to the data points. The current output is then obtained from the time derivative of the charge curve. Fig. 4 shows the induced charge and corresponding current output obtained from the simulation.
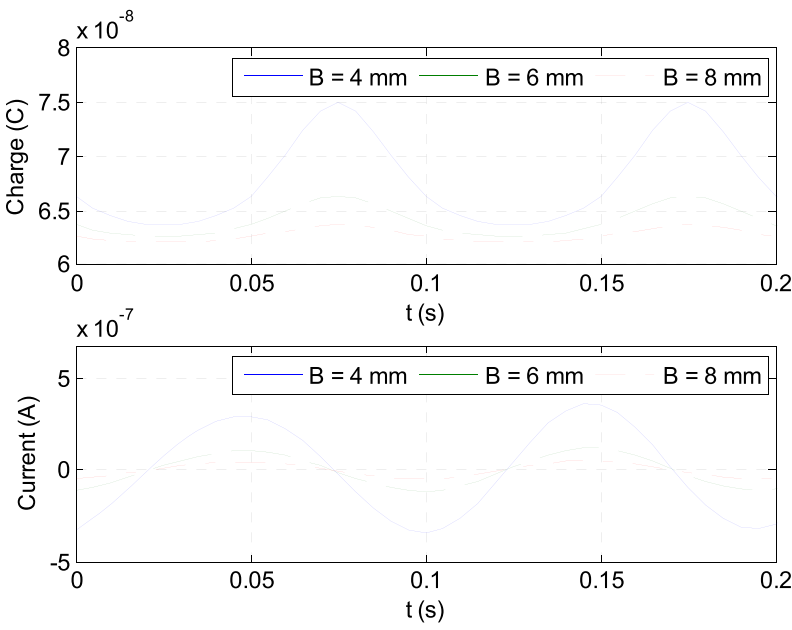

Fig. 5. Induced charge and current output for different center positions.

A comparison between Fig. 2 and Fig. 4 indicates that the induced charge is dependent on the transverse distance between the electrode and the belt. The approaching of the belt to the electrode leads to more induced charge, whereas less induced charge is generated when the belt moves away. The cyclic variation of the induced charge, due to the belt vibration, gives rise to an alternating current output flowing periodically into and out of the electrode. In order to shed light on how the sensor signal is related to the belt vibration, the sensing characteristics of the electrostatic sensor are investigated.

\section{Sensing Characteristics}

With the assumption that the charges are uniformly distributed on the belt surface and in view of the superposition principle of electrostatic field, it is reasonable to study the sensing characteristics of the electrostatic sensor using a point charge vibrating harmonically in the normal direction of the electrode surface. In this second simulation, the center of the electrode is located at the origin of the coordinate system. A point charge, carrying net electrostatic charges of $-1 \mu \mathrm{C}$, is required to move along the $\mathrm{z}$-axis according to the following equation

$$
z(t)=B+A \sin (2 \pi f t)
$$

where $B$ is the center position of the vibration, $A$ denotes the vibration amplitude and $f$ represents the vibration frequency.

The induced charge and current output for different center positions are first examined. Fig. 5 shows the simulation results when the vibration amplitude is $2 \mathrm{~mm}$ and the vibration frequency is $10 \mathrm{~Hz}$. It is apparent that the frequency of the current output is identical to the vibration frequency of the point charge. A shorter distance between the center position of the point charge and the electrode yields a higher current output, suggesting that the electrode should be placed closer to the belt in order to obtain a stronger sensor signal. It is also noticeable that the fluctuation of the induced charge is unsymmetrical about the value obtained when the point charge is at the center position. As a result, the current output is also unsymmetrical about the zero value and in time. The difference 


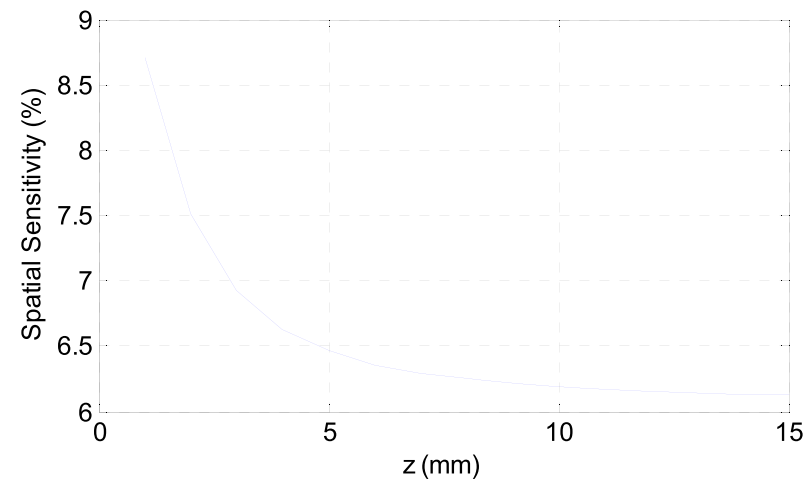

Fig. 6. Spatial sensitivity along the z-axis.

between the maximum current flowing into and out of the electrode becomes larger as the electrode is placed closer to the belt. The above findings can be attributed to the non-uniform spatial sensitivity of the electrostatic sensor, which is defined as the ratio of the induced charge to the source charge. Fig. 6 shows the spatial sensitivity of the electrostatic sensor along the z-axis. As expected, the sensor is more sensitive to nearby charges, but the spatial sensitivity becomes more uniform at farther locations.

In order to quantify the effects of non-uniform spatial sensitivity on the distortion of the sensor signal, the spectra of the current outputs for different center positions are plotted in Fig. 7. The length of the signal is $6 \mathrm{~s}$ for spectrum calculation. As illustrated, the distortion produces higher order harmonics that are integer (whole number) multiples of the fundamental frequency. The Total Harmonic Distortion $(T H D)$, is defined as the ratio of the RMS amplitude of a set of higher order harmonic frequencies to the RMS amplitude of the fundamental frequency:

$$
T H D=\frac{\sqrt{A_{2}^{2}+A_{3}^{2}+A_{4}^{2}+\cdots}}{A_{1}}
$$

where $A_{i}$ is the RMS amplitude of the $i$-th harmonic. Omitting the fourth and higher order harmonics that have negligible amplitudes, the values of $T H D$ for center positions of $4 \mathrm{~mm}$, $6 \mathrm{~mm}$ and $8 \mathrm{~mm}$ are calculated as $0.595,0.443$ and 0.362 , respectively. It follows from the above results that a relatively larger distance between the electrode and the belt reduces the distortion of the sensor signal. From a practical viewpoint, if the electrode is placed too close to the belt, the risk of direct contact increases, which may damage the sensor and give rise to an erroneous signal.

Fig. 8 shows the induced charge and the current output for different vibration amplitudes, when the center position is $6 \mathrm{~mm}$ and the vibration frequency is $10 \mathrm{~Hz}$. As illustrated, the electrode delivers more current output at a larger vibration amplitude, under which a longer excursion distance is covered and thus the variation of induced charge is more intense. It is also believed that for the same vibration frequency, an increased vibration amplitude is associated with a higher transverse velocity of the belt, which determines the rate of change of the induced charge (literally the current).

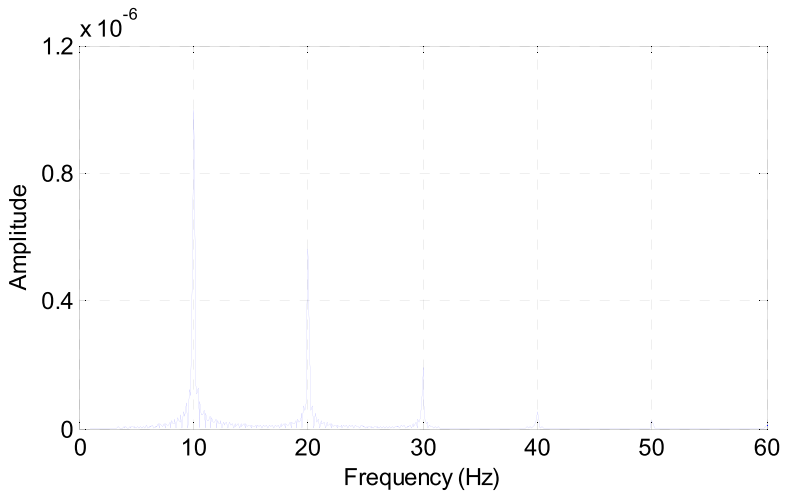

(a)

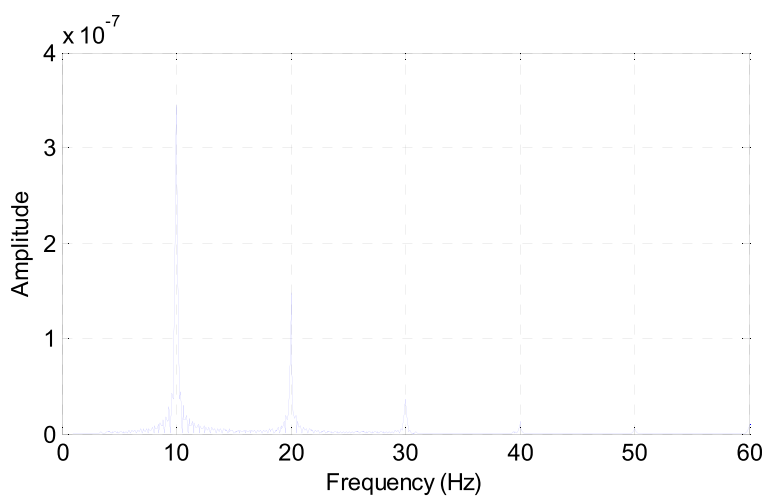

(b)

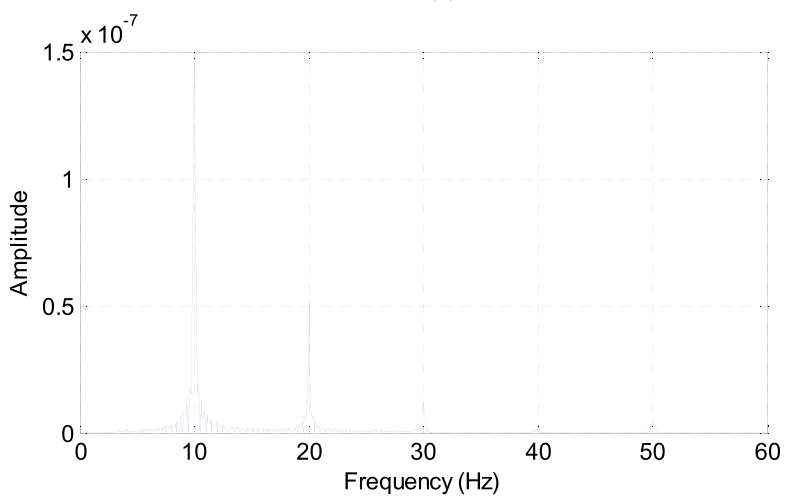

(c)

Fig. 7. Amplitude spectra of the current outputs for different center positions. (a) Center position $4 \mathrm{~mm}$. (b) Center position $6 \mathrm{~mm}$. (c) Center position $8 \mathrm{~mm}$.

In what follows, the influence of transverse velocity on the current output will be examined by varying the vibration frequency.

The induced charge and the current output for different vibration frequencies are plotted in Fig. 9, with the center position being $6 \mathrm{~mm}$ and the vibration amplitude $2 \mathrm{~mm}$. It is observed that the amplitude of the alternating current output increases with the vibration frequency. Therefore, the above conjecture that the belt transverse velocity affects the current output can be proved. If the variation of induced charge can be approximated using a sinusoidal function, it is easy to understand, from a mathematical point of view, that the amplitude of its time derivative (the current) is proportional to the frequency. 


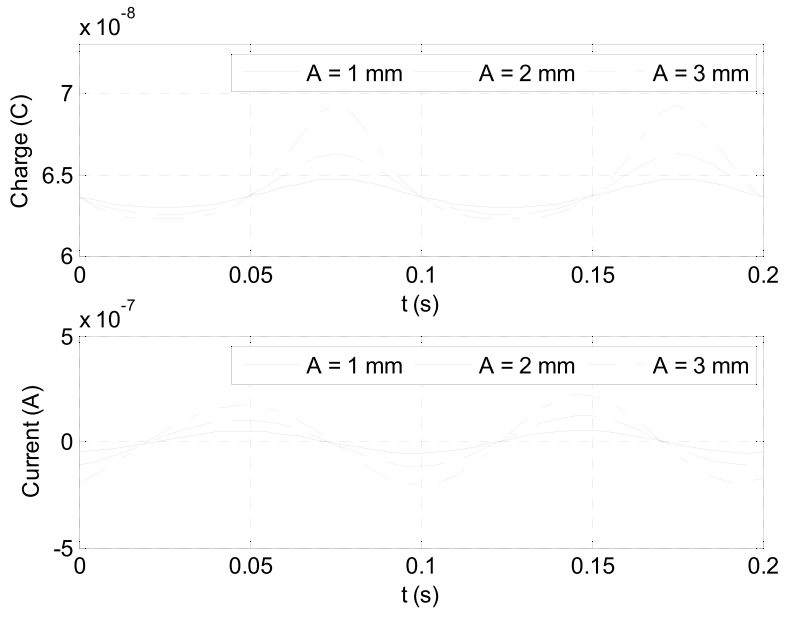

Fig. 8. Induced charge and current output for different vibration amplitudes.
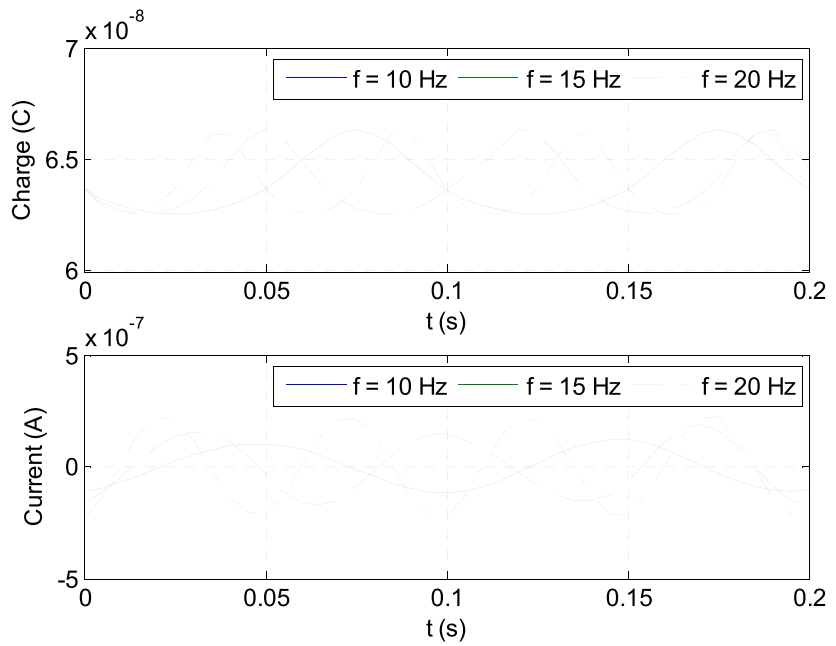

Fig. 9. Induced charge and current output for different vibration frequencies.

\section{Measurement Method}

The simulation results illustrate that the sensor signal is strongest when the point charge is passing through the equilibrium position and zero at the upper and lower maximumdisplacement limits. Therefore, the sensor signal reflects the transverse velocity of the belt. However, the absolute value of the transverse velocity cannot be measured using the electrostatic sensor due to the vulnerability of the electrostatic sensing technique to environmental and operating conditions [27]. It is also worth noting that the transverse velocity is positively, but nonlinearly related to the sensor signal due to the non-uniform spatial sensitivity of the sensor.

As indicated by equation (3), the belt vibrates at the fundamental frequency as well as the higher order harmonics. Although the distortion due to the non-uniform spatial sensitivity also produces higher order harmonics, its influence can be effectively minimized if the electrode is placed adequately away from the belt, as observed in Fig. 7. In order to verify that the belt vibration can be characterized through spectral analysis of the sensor signal, the sensor response to a multimode vibration is compared to that of a single-mode vibration. The following equation describes the kinematics of a point

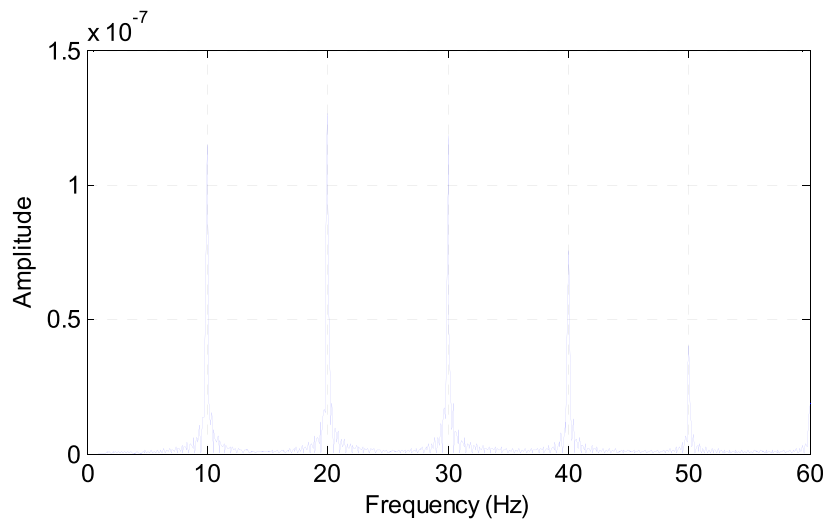

Fig. 10. Amplitude spectra of the current output for a multi-mode vibration (signal length $6 \mathrm{~s}$ ).

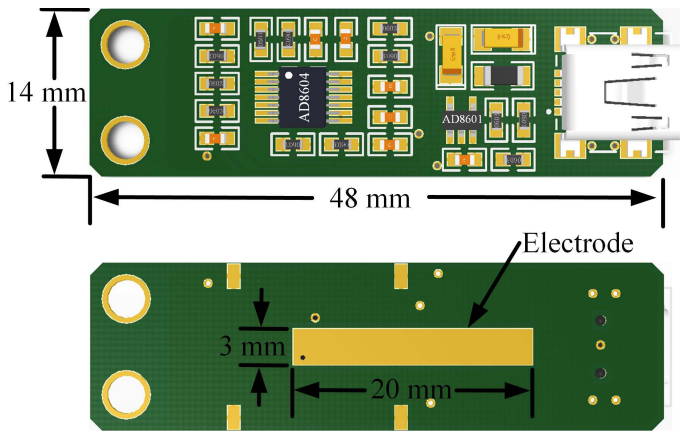

Fig. 11. Electrostatic sensor

charge vibrating in three modes simultaneously:

$$
z(t)=8+2 \sin (20 \pi t)+\sin (40 \pi t)+0.5 \sin (60 \pi t)
$$

The spectrum of the current output for this multi-mode vibration is shown in Fig. 10. Compared to Fig. 7(c) that depicts the signal spectrum for the first vibration mode, the second and third order harmonics become stronger than the fundamental frequency. It therefore proves feasible to monitor higher order vibration modes using the electrostatic sensor. Although the fourth and fifth order harmonics also become stronger due to distortion, their magnitudes are not strong enough to support the presence of the corresponding vibration modes.

\section{EXPERIMENTAL RESUlTS AND DISCUSSION}

\section{A. Experimental Setup}

The physical instantiation of the electrostatic sensor is a two-layer printed circuit board with the electrode residing on the bottom layer as a long pad. On the top layer is the signal conditioning circuit consisting of five consecutive stages built with wideband rail-to-rail operational amplifiers of AD8604 and AD8601. The first stage is the I-V converter that outputs a small voltage signal. The second stage amplifies the signal using a non-inverting amplifier. A Salley-Key low-pass filter at the third stage eliminates high frequency noises. The signal is further amplified at the fourth stage and then level shifted from a bipolar signal to a unipolar signal at the final stage for subsequent sampling. Fig. 11 shows the electrostatic sensor with the dimensions labelled. 


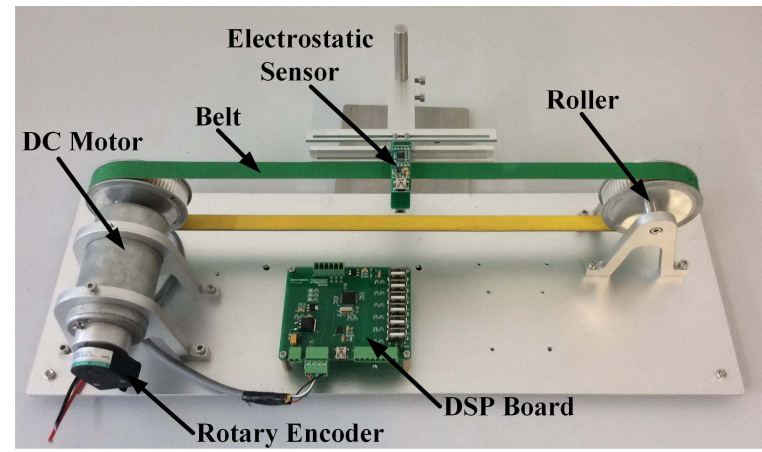

Fig. 12. Test rig.

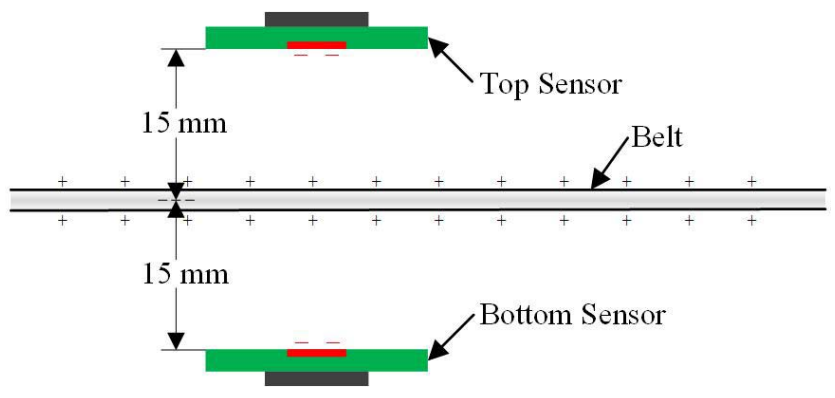

Fig. 13. Sensing arrangement.

Experiments with the electrostatic sensor were undertaken on a purpose-built test rig, as shown in Fig. 12. A flat, nylon-type belt is stretched over two identical pulleys, with one pulley being actuated by a DC motor. The running speed of the belt can be adjusted from 0 to $10 \mathrm{~m} / \mathrm{s}$ by regulating the voltage of the motor. The characteristics of the belt vibration that is naturally generated vary with the running speed. The electrostatic sensor is mounted on a supporting frame that allows the distance between the electrode and belt to be adjusted. A DSP board performs sampling and on-line processing of the sensor signal, and measures the motor rotational speed using a photoelectric rotary encoder as well.

\section{B. Sensor Signals}

In order to validate the supposition that the fluctuation of the sensor signal is primarily caused by the belt vibration, two electrostatic sensors placed on both sides of the belt are employed to monitor the belt motion simultaneously. Fig. 13 shows the sensing arrangement, in which the belt runs between two electrodes separated by $30 \mathrm{~mm}$. Fig. 14 depicts the electrostatic signals collected when the belt running speed is $2.0 \mathrm{~m} / \mathrm{s}$. It can be noticed that the two signals exhibit nearly anti-phasing oscillatory behaviors, which follows that an increase of the induced charge on one electrode due to belt approaching corresponds to a reduction on the other electrode. The amplitude spectra of the signals are plotted in Fig. 15. It is clear that the two spectra are very alike, with the same dominant vibration modes occurring at several distinct frequencies below $100 \mathrm{~Hz}$. The spectral peaks at exactly $50 \mathrm{~Hz}$ are believed to be caused by the power line interference.

\section{Measurement Results}

The vibration dynamics of a travelling belt is determined by a variety of factors, including belt tension, axial speed,

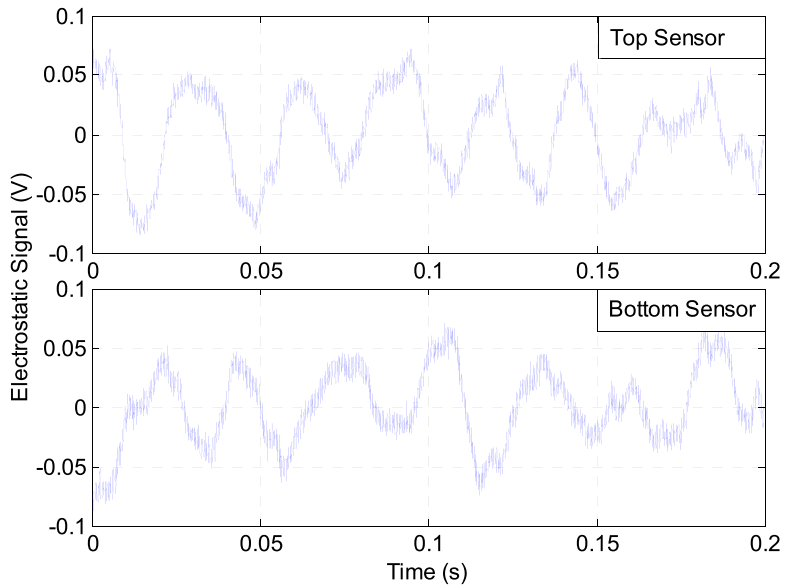

Fig. 14. Electrostatic signals collected on both sides of the belt.

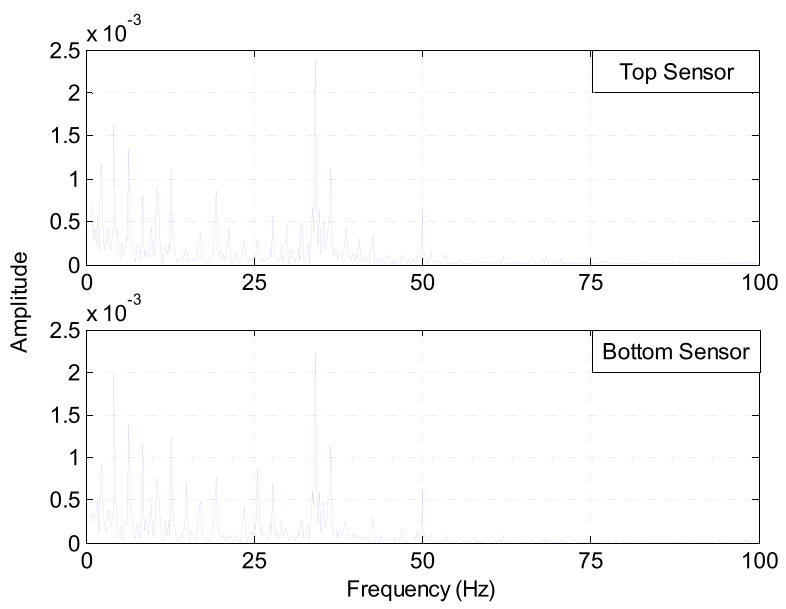

Fig. 15. Amplitude spectra of the electrostatic signals in Fig. 14.

span length, viscoelastic stiffness and various mechanical abnormalities as described in [1]. In order to assess the measurement performance of the electrostatic sensor, the belt axial speed was varied and the vibration at the middle point of the belt span was monitored. Fig. 16 shows the amplitude spectra for different speeds when the distance between the sensor and the belt is $15 \mathrm{~mm}$. As can be seen, there exist strong, wellseparated spectral peaks located at the fundamental frequency and its higher order harmonics. Since the sensor is placed far enough from the belt and the magnitudes of higher order harmonics are much stronger than that of the fundamental frequency, the influence of signal distortion can be neglected and the harmonics identified as the vibration modes. As indicated in Fig.16, the vibration frequencies increase linearly with the belt axial speed. Fig.16 also shows that the amplitudes of the spectral peaks become stronger at a faster speed, suggesting an increase of the transverse velocity of the belt. However, a higher belt axial speed gives rise to more charges on the belt, which also leads to elevated signal strength. Although the contributions of both factors cannot be quantified, the vibration frequencies and the relative magnitude of the spectral peaks provide adequate prognostic state awareness. In addition, there exists a prominent spectral peak between the 4th and 5th order harmonics. Since the frequency of this peak is exactly the rotational frequency of the pulley, it is believed that some 


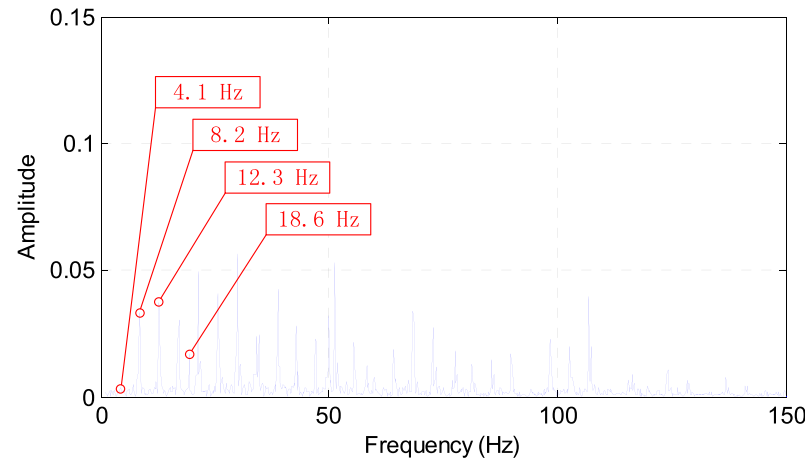

(a)

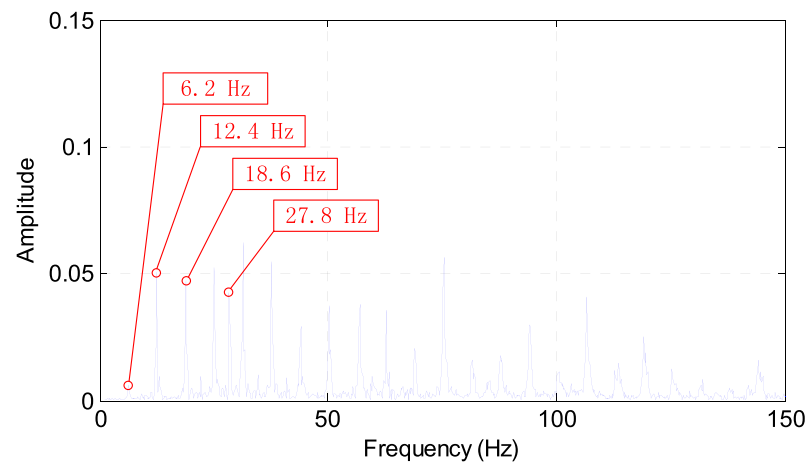

(b)

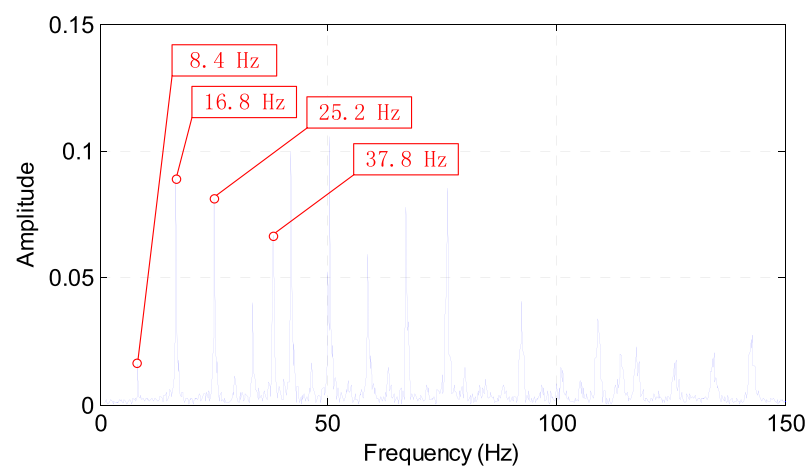

(c)

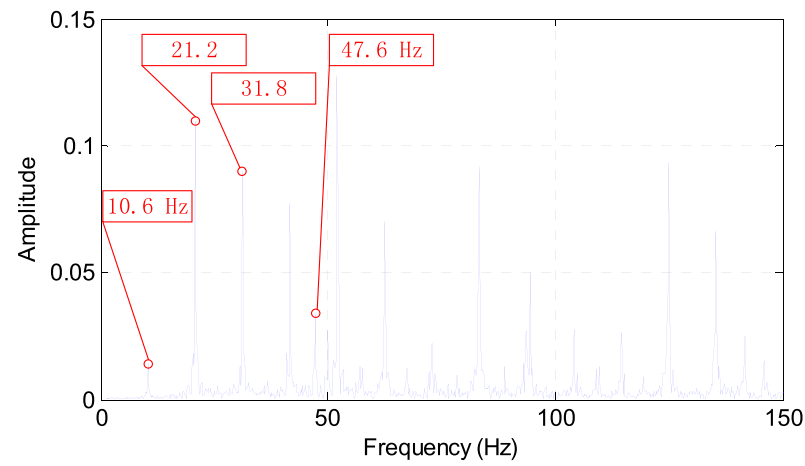

(d)

Fig. 16. Amplitude spectra for different speeds when the distance between the sensor and the belt is $15 \mathrm{~mm}$. (a) $4 \mathrm{~m} / \mathrm{s}$. (b) $6 \mathrm{~m} / \mathrm{s}$. (c) $8 \mathrm{~m} / \mathrm{s}$. (d) $10 \mathrm{~m} / \mathrm{s}$.

manufacturing or assembly imperfection associated with the pulley, such as eccentricity and misalignment, has led to this abnormal vibration.

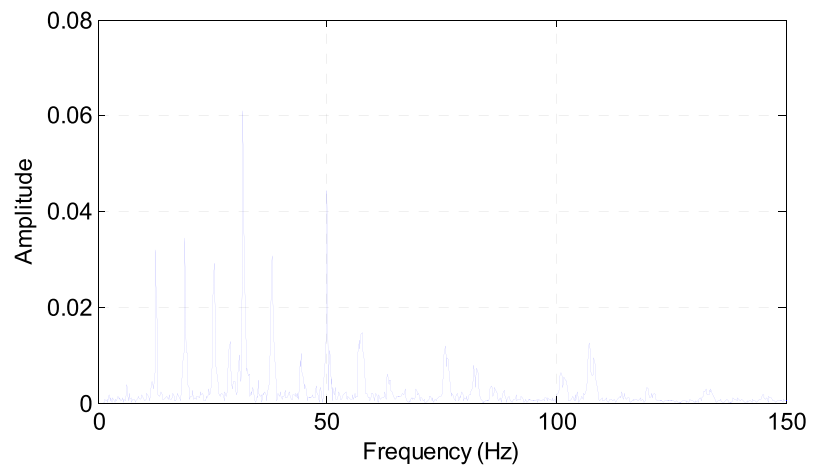

(a)

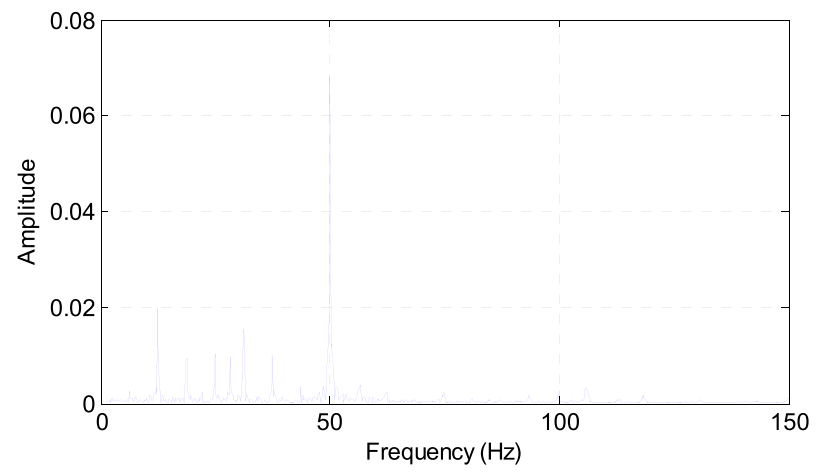

(b)

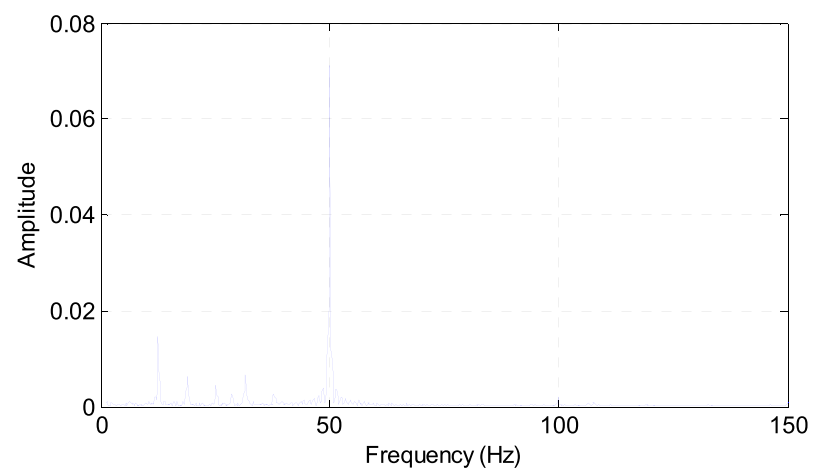

(c)

Fig. 17. Amplitude spectra for different distances between the electrode and the belt for a constant belt speed of $6 \mathrm{~m} / \mathrm{s}$. (a) $25 \mathrm{~mm}$. (b) $45 \mathrm{~mm}$. (c) $65 \mathrm{~mm}$.

As discussed above, the distance between the electrode and the belt affects the performance of the system. To assess its effect, the distance was varied from $15 \mathrm{~mm}$ to $65 \mathrm{~mm}$ for a constant belt speed of $6 \mathrm{~m} / \mathrm{s}$. Fig. 17 shows the amplitude spectra for distances of $25 \mathrm{~mm}, 45 \mathrm{~mm}$ and $65 \mathrm{~mm}$, respectively, while the result for $15 \mathrm{~mm}$ is depicted in Fig. 16 (b). As can be seen, the strength of the electrostatic signal decreases and the $50 \mathrm{~Hz}$ power line interference becomes more perceptible as the electrode is moved away from the belt. The vibration modes that can be detected also vary with the distance, i.e. the higher order harmonics diminish or disappear for a longer distance. According to the theory of mechanical vibration [32], the total transverse displacement of the belt is given by the superposition of all modes. Although the transverse velocity of a higher order vibration mode is greater, its displacement is smaller than that of lower order modes. 
Therefore, when the electrode is far away, the variation of induced charge due to the minute vibration displacement of a higher order mode is very small. By contrast, a lower order mode can perform large-amplitude deflection in the sensing zone, thus leading to the increased relative magnitude in the spectra. It is worth noting that an adequately longer distance between the sensor and the belt alleviates the influence of distortion caused by non-uniform spatial sensitivity, which also reduces the relative magnitudes of the higher order harmonics.

\section{Conclusions}

A novel measurement technique based on electrostatic sensing has been presented in this paper for on-line vibration monitoring of power transmission belts. The response of a strip-shaped electrode to a belt moving both axially and transversely has been studied through finite element modelling. A measurement method of belt vibration has been developed based on investigations into the sensor's sensing characteristics. Performance assessment of the measurement technique has been undertaken on a two-pulley belt drive system. The electrostatic signals collected from sensors on both sides of the belt have validated that the signal fluctuations are dominated by the transverse belt vibration. In addition, the belt vibrations at different axial speeds have been measured, which shows that the resonant frequencies and transverse velocity increase with the axial speed. The signal strength and the number of vibration modes identifiable increase for a closer distance between the electrode and the belt.

In future work, the effects of belt type, material property and surface roughness on the performance of the measurement system will be examined under a wider range of environmental conditions. Methods of compensation for the distortion due to the non-uniform spatial sensitivity will be explored for enhanced measurement accuracy. Applications of the system to fault diagnosis and prognosis of industrial belt drive systems will also be carried out. Practical issues, such as electromagnetic interferences and vibration of the sensor itself due to mechanical connection to the machine will be addressed.

\section{REFERENCES}

[1] H. Yamashina, S. Okumura, and I. Kawai, "Development of a diagnosis technique for failures of V-belts by a cross-spectrum method and a discriminant function approach," J. Intell. Manuf., vol. 7, no. 1, pp. 85-93, 1996.

[2] J. R. Coombes and Y. Yan, "Experimental investigations into the flow characteristics of pneumatically conveyed biomass particles using an electrostatic sensor array," Fuel, vol. 151, pp. 11-20, Jul. 2015.

[3] X. Qian, X. Huang, H. Yonghui, and Y. Yan, "Pulverized coal flow metering on a full-scale power plant using electrostatic sensor arrays," Flow Meas. Instrum., vol. 40, pp. 185-191, Dec. 2014.

[4] C. He, X. T. Bi, and J. R. Grace, "Simultaneous measurements of particle charge density and bubble properties in gas-solid fluidized beds by dualtip electrostatic probes," Chem. Eng. Sci., vol. 123, pp. 11-21, Feb. 2015.

[5] W. Zhang, Y. Cheng, C. Wang, W. Yang, and C.-H. Wang, "Investigation on hydrodynamics of triple-bed combined circulating fluidized bed using electrostatic sensor and electrical capacitance tomography," Ind. Eng. Chem. Res., vol. 52, no. 32, pp. 11198-11207, 2013.

[6] T. Addabbo, A. Fort, R. Garbin, M. Mugnaini, S. Rocchi, and V. Vignoli, "Theoretical characterization of a gas path debris detection monitoring system based on electrostatic sensors and charge amplifiers," Measurement, vol. 64, pp. 138-146, Mar. 2015.
[7] Z. Wen, X. Ma, and H. Zuo, "Characteristics analysis and experiment verification of electrostatic sensor for aero-engine exhaust gas monitoring," Measurement, vol. 47, pp. 633-644, Jan. 2014.

[8] T. J. Harvey, R. J. K. Wood, and H. E. G. Powrie, "Electrostatic wear monitoring of rolling element bearings," Wear, vol. 263, nos. 7-12, pp. 1492-1501, 2007.

[9] L. Wang, Y. Yan, Y. Hu, and X. Qian, "Rotational speed measurement using single and dual electrostatic sensors," IEEE Sensors J., vol. 15, no. 3, pp. 1784-1793, Mar. 2015.

[10] Y. Yan, S. J. Rodrigues, and Z. Xie, "Non-contact strip speed measurement using electrostatic sensing and correlation signal-processing techniques," Meas. Sci. Technol., vol. 22, no. 7, p. 075103, 2011.

[11] Y. Hu, Y. Yan, L. Wang, X. Qian, and X. Wang, "Simultaneous measurement of belt speed and vibration through electrostatic sensing and data fusion," IEEE Trans. Instrum. Meas., doi: 10.1109/TIM.2015.2490958.

[12] S. Abrate, "Vibrations of belts and belt drives," Mech. Mach. Theory, vol. 27 , no. 6, pp. 645-659, 1992.

[13] L.-Q. Chen, "Analysis and control of transverse vibrations of axially moving strings," Appl. Mech. Rev., vol. 58, no. 2, pp. 91-116, 2005.

[14] R. A. Malookani and W. T. van Horssen, "On resonances and the applicability of Galerkin's truncation method for an axially moving string with time-varying velocity," J. Sound Vib., vol. 334, pp. 1-17, May 2015.

[15] H. Zhu, Y. Hu, and Y. Pi, "Transverse hysteretic damping characteristics of a serpentine belt: Modeling and experimental investigation," J. Sound Vib., vol. 333, no. 25, pp. 7019-7035, 2014.

[16] X.-D. Yang and W. Zhang, "Nonlinear dynamics of axially moving beam with coupled longitudinal-transversal vibrations," Nonlinear Dyn., vol. 78, no. 4, pp. 2547-2556, 2014.

[17] B. B. Özhan, "Vibration and stability analysis of axially moving beams with variable speed and axial force," Int. J. Struct. Stability Dyn., vol. 14 no. 6, p. 1450015, 2014.

[18] M. Amar, I. Gondal, and C. Wilson, "Vibration spectrum imag ing: A novel bearing fault classification approach," IEEE Trans. Ind. Electron., vol. 62, no. 1, pp. 494-502, Jan. 2015.

[19] K. Javed, R. Gouriveau, N. Zerhouni, and P. Nectoux, "Enabling health monitoring approach based on vibration data for accurate prognostics," IEEE Trans. Ind. Electron., vol. 62, no. 1, pp. 647-656, Jan. 2015.

[20] W. Qiao and D. Lu, "A survey on wind turbine condition monitoring and fault diagnosis-Part II: Signals and signal processing methods," IEEE Trans. Ind. Electron., vol. 62, no. 10, pp. 6546-6557, Oct. 2015.

[21] H. Chaurasiya, "Recent trends of measurement and development of vibration sensors," Int. J. Comput. Sci. Issues, vol. 9, no. 4, pp. 1-6, 2012.

[22] R. Atashkhooei, J.-C. Urresty, S. Royo, J.-R. Riba, and L. Romeral, "Runout tracking in electric motors using self-mixing interferometry," IEEE/ASME Trans. Mechatronics, vol. 19, no. 1, pp. 184-190, Feb. 2014.

[23] P. Catalano, F. Fucci, F. Giametta, G. La Fianza, and B. Bianchi, "Vibration analysis using a contactless acquisition system," Proc. SPIE, vol. 8881, p. 888108, May 2013.

[24] R. D. Sante and G. L. Rossi, "A new approach to the measurement of transverse vibration and acoustic radiation of automotive belts using laser Doppler vibrometry and acoustic intensity techniques," Meas. Sci. Technol., vol. 12, no. 4, pp. 525-533, 2001.

[25] A. Agnani, M. Martarelli, and E. P. Tomasini, "V-belt transverse vibration measurement by means of laser Doppler vibrometry," Proc. SPIE, vol. 7098, p. 709819, Jun. 2008.

[26] J. Moon and J. A. Wickert, "Non-linear vibration of power transmission belts," J. Sound Vib., vol. 200, no. 4, pp. 419-431, 1996.

[27] S. H. S. Javadi and D. H. Napier, "Electrification of dielectric belts running on earthed metal rollers," in Proc. IChemE Symp. Series, vol. 39a. Apr. 1974, pp. 266-279.

[28] S. Franco, Design With Operational Amplifiers and Analog Integrated Circuits, 4th ed. New York, NY, USA: McGraw-Hill, 2014.

[29] E. M. Purcell and D. J. Morin, Electricity and Magnetism, 3rd ed. Cambridge, U.K.: Cambridge Univ. Press, 2013.

[30] L. Wang and Y. Yan, "Mathematical modelling and experimental validation of electrostatic sensors for rotational speed measurement," Meas. Sci. Technol., vol. 25, no. 11, p. 115101, 2014.

[31] A. Vermeer and B. A. Strasters, "The charge transport in an electrostatic belt generator," Nucl. Instrum. Methods, vol. 131, pp. 213-222, Dec. 1975.

[32] S. S. Rao, Mechanical Vibrations, 5th ed. Upper Saddle River, NJ, USA: Prentice-Hall, 2011. 


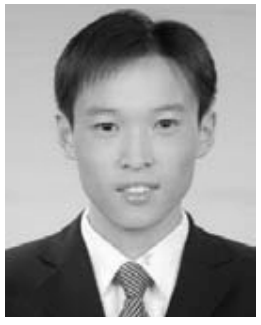

Yonghui Hu received the B.Eng. degree in automation from the Beijing Institute of Technology, Beijing, and the Ph.D. degree in dynamics and control from Peking University, Beijing, in 2004 and 2009, respectively.

He was a Post-Doctoral Research Fellow with Beihang University, Beijing, from 2010 to 2012. $\mathrm{He}$ is currently a Lecturer with the School of Control and Computer Engineering, North China Electric Power University, Beijing. His current research interests include measurement of multiphase flow and condition monitoring of mechanical systems.

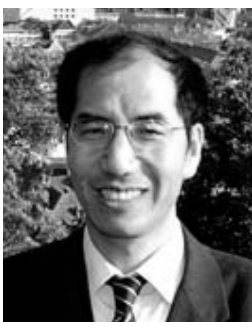

Yong Yan (M'04-SM'04-F'11) received the B.Eng. and M.Sc. degrees in instrumentation and control engineering from Tsinghua University, Beijing, China, in 1985 and 1988, respectively, and the Ph.D. degree in flow measurement and instrumentation from the University of Teesside, Middlesbrough, U.K., in 1992.

He was an Assistant Lecturer with Tsinghua University in 1988. He joined the University of Teesside in 1989, as a Research Assistant. After a short period of post-doctoral research, he was a Lecturer with the University of Teesside from 1993 to 1996, and then as a Senior Lecturer, Reader, and Professor with the University of Greenwich, Chatham, U.K., from 1996 to 2004. He is currently a Professor of Electronic Instrumentation, the Head of the Instrumentation, Control and Embedded Systems Research Group, and the Director of Research with the School of Engineering and Digital Arts, University of Kent, Canterbury, U.K. He has authored in excess of 300 research papers in journals and conference proceedings with an h-index of 33 .

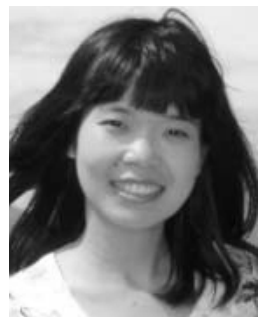

Lijuan Wang received the B.Eng. degree in computer science and technology from Qiqihar University, Qiqihar, China, in 2010, and the Ph.D. degree in measurement and automation from North China Electric Power University, Beijing, China, in 2015.

She is currently involved in post-doctoral research in instrumentation and measurement with North China Electric Power University. Her current research interests include electrostatic sensing, flow measurement, and digital signal processing.

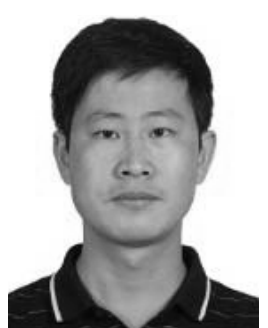

Xiangchen Qian received the B.Eng. degree in automation from the Tianjin University of Technology, Tianjin, China, in 2004, the M.Sc. degree in automatic meter and device from Tianjin University, Tianjin, in 2007, and the Ph.D. degree in electronics engineering from the University of Kent, Canterbury, U.K., in 2013.

$\mathrm{He}$ is currently a Lecturer with the School of Control and Computer Engineering, North China

Electric Power University, Beijing, China. His current research interests include multiphase flow measurement techniques, development of instrumentation systems, and digital signal processing. 